\title{
An improved differential evolution algorithm to parameters estimation of Volterra digital systems
}

\author{
Wei-Der Chang ${ }^{*}$ Shan-Cheng Pan Yu-Rong Chiu \\ Department of Computer and Communication, Shu-Te University \\ Kaohsiung 824, Taiwan
}

*Corresponding Author: wdchang@ @stu.edu.tw

\begin{abstract}
This paper will present a new parameter estimation method for Volterra digital systems by an improved differential evolution (DE) algorithm. The DE algorithm has been proven to be an efficient means to solve a variety of engineering optimization problems. To further enhance the searching capacity, the chaotic random number produced by the logistic map system is incorporated into the algorithm to replace the mutation constant factor. Based on the improved DE algorithm, the parameter estimation design problem for the Volterra digital systems is considered and solved. Some simulation results will reveal that the proposed design method can exactly solve for all the system parameters.
\end{abstract}

Keywords: improved differential evolution, Logistic map system, chaotic random number, Volterra digital systems.

\section{Introduction}

Volterra digital system is one of nonlinear digital systems and basically it is an extended version of the nonrecursive digital filter (or called the finite impulse response (FIR) filter). The new output of Volterra digital system is influenced by the present and past input signals and their cross-product terms, not influenced by the past output signals. In recent years, a number of researches concerned with Volterra digital systems have been reported $^{(1-5)}$. In [1], the authors presented an efficient nonparametric time domain nonlinear system identification method based on truncated Volterra series models. In [3], the pipelined second-order adaptive Volterra filter was extended into the high order version, and the pipelined adaptive Volterra set-membership algorithm was proposed to reduce the computational complexity. Simulations verify the performance of the proposed algorithms under Gaussian noise and impulsive noise environments. Moreover, the authors developed a Volterra series approximation approach using the adaptive filter concept for nonlinear identification of multi-degree of freedom systems ${ }^{(4)}$. The effectiveness of the proposed scheme was confirmed by testing some systems.

The design scheme for solving the parameter estimation problem of Volterra digital system here is the differential evolution (DE) algorithm which has been proven to be an effective and powerful means to various optimization problems, for instances, network traffic forecasting ${ }^{(6)}$, congestion management ${ }^{(7)}$, exergoeconomic analysis and optimization of a cogeneration system $^{(8)}$, workload prediction in cloud ${ }^{(9)}$, and community detection ${ }^{(10)}$. In our previous work ${ }^{(11)}$, the classical DE algorithm was used to successfully solve for parameter estimation of bilinear digital filters. Like those of genetic algorithm, the DE is composed of three evolutionary operations including mutation, crossover, and selection to achieve the optimization purpose. Also, the DE is a population-based algorithm with multiple direction searching. In this study, an improved DE algorithm with chaotic random numbers is utilized to enhance the searching ability. The chaotic system to generate a series of random numbers is the logistic map which is a very simple system with one control parameter ${ }^{(12)}$. The remainder of this paper can be summarized as follows. Section 2 will give some descriptions of Volterra digital systems. In Section 3, the improved DE algorithm with chaotic random numbers is introduced in detail, and the logistic map chaotic system is 
clearly described as well. In Section 4, based on the proposed method, the parameter estimation problem of Vloterra digital system is illustrated. Some simulation results are further given to verify the feasibility of the proposed method. Finally, Section 5 will address a simple conclusion and the future possible work.

\section{Volterra digital systems}

The architecture of the digital filter can be generally divided into two main categories: the finite impulse response (FIR) filter and the infinite impulse response (IIR) filter. The new output of FIR filter is generated by the present and past input signals, and the IIR output is also influenced by the past output signals besides the present and past input signals. In general, the FIR digital filter can be described by the following difference equation

$$
\begin{aligned}
y[n] & =\sum_{k=0}^{M} h[k] x[n-k] \\
& =h[0] x[n]+h[1] x[n-1]+\cdots+h[M] x[n-M],
\end{aligned}
$$

where $x$ and $y$ represent the input and output signals, respectively, $h$ denotes the system parameter of the digital filter, $M$ is then the number of past input signals required. In this study, we will focus on the parameter estimation of the Volterra digital system that is basically an extended version of FIR structure. Eq. (2) shows its dynamical difference equation

$$
\begin{aligned}
& y[n]=h[0]+\sum_{k=1}^{M} h[k] x[n-k+1] \\
& +\sum_{k_{1}=0}^{M-1} \sum_{k_{2}=k_{1}}^{M-1} h\left[k_{1}, k_{2}\right] x\left[n-k_{1}\right] x\left[n-k_{2}\right] \\
& =h[0]+h[1] x[n]+\cdots+h[M] x[n-M+1] \\
& +h[0,0] x^{2}[n]+\cdots+h[0, M-1] x[n] x[n-M+1] \\
& +h[1,1] x^{2}[n-1]+\cdots+h[M-1, M-1] x^{2}[n-M+1],
\end{aligned}
$$

where $h\left[k_{1}, k_{2}\right]$ means the second-order system parameter to reflect the quadratic properties of such the digital system. It is expected that all the system parameters, $h[k]$ and $h\left[k_{1}, k_{2}\right]$, involved in the Volterra digital system can be exactly estimated and identified by the proposed design method. In order to introduce the DE algorithm into the parameter estimation of Volterra digital filter, we further rewrite Eq. (2) to be a new vector form as follows

$$
y[n]=\Theta X^{T},
$$

where $X$ is the vector of collecting the present, past, and their cross-product terms as

$$
X=\left[1, x[n], \cdots, x[n-M+1], x^{2}[n],\right.
$$

$$
\left.x[n] x[n-1], \cdots, x^{2}[n-M+1]\right],
$$

and $\Theta$ is a collection of all the system parameters given by

$$
\begin{aligned}
\Theta & =\left[\theta_{1}, \theta_{2}, \cdots, \theta_{L}\right] \\
& =[h[0], h[1], \cdots, h[M], h[0,0], h[0,1] \cdots, \\
& h[M-1, M-1]],
\end{aligned}
$$

where the subscript $L$ then represents the number of system parameters estimated. The parameter vector of Eq. (5) will be utilized in the DE algorithm. Many such vectors further form a population of the algorithm, and some evolving mechanisms are performed on this population in order to obtain better offspring.

\section{Modified DE algorithm with chaotic random numbers}

In this study, we attempt to use the modified DE algorithm to identify the system parameters of the Volterra digital filter. Different from the general $\mathrm{DE}$, in the proposed version the mutation constant factor used in the algorithm is replaced by a series of chaotic random numbers that is produced by the logistic map chaotic system. The general DE contains three evolutionary mechanisms: mutation, crossover, and selection to achieve the optimization. Before introducing the detailed algorithm, a cost function for evaluating the performance of parameter vector should be defined in advance, and here it is given by Eq. (6)

$$
C F=\sum_{n=0}^{N} e^{2}[n]=\sum_{n=0}^{N}[y[n]-\hat{y}[n]]^{2},
$$

where $N$ is the sampling number assigned by the designer and $e$ is the error signal between the actual output $y$ and the model output $\hat{y}$. To solve the parameter estimation problem, it is a better parameter vector if its corresponding cost function is fewer. The following is the detailed description for the DE algorithm ${ }^{(5)}$. 
- Mutation operation

The nutation operation is to generate a new mutated vector $V=\left[v_{1}, v_{2}, \cdots v_{L}\right]$ according to Eq. (7)

$V=\Theta_{\alpha}+F\left(\Theta_{\beta}-\Theta_{\gamma}\right)$,

where $\Theta_{\alpha}, \Theta_{\beta}$, and $\Theta_{\gamma}$ are three different parameter vectors randomly selected from the population and $F$ is a mutation constant factor that controls the amplification of the differential variation $\Theta_{\beta}-\Theta_{\gamma}$, it is a constant value in the general DE algorithm. It is clearly seen from Eq. (7) that the mutated vector $V$ is a combination of mutating parameters $\Theta_{\alpha}, \Theta_{\beta}$, and $\Theta_{\gamma}$. In this paper, the chaotic random number is employed to substitute for the fixed mutation factor. To generate a sequence of chaotic random numbers, a logistic map system is adopted and its dynamic equation is simply expressed by ${ }^{(12)}$

$$
y[n]=\mu y[n-1](1-y[n-1])
$$

where $0 \leq \mu \leq 4$ is the system control parameter. Eq. (8) will display chaotic dynamics when $\mu=4$ and $y[0] \notin\{0,0.25,0.5,0.75,1\}$, and the output $y[n]$ is distributed in the interval $(0,1)$ provided that the initial value $y[0] \in(0,1)$. Fig. 1 shows a sequence of chaotic random numbers when $\mu=4$ and $y[0]=0.3$. This chaotic sequence is employed to replace the fixed mutation constant in the following simulation.



Fig. 1. Output of logistic map system with $\mu=4$ and $y[0]=0.3$.

\section{- Crossover operation}

This mutated vector $V$ further cross with a target vector $\Theta=\left[\theta_{1}, \theta_{2}, \cdots, \theta_{L}\right]$ in the crossover operation. It means that some elements between the mutated vector $V$ and the target vector $\Theta$ are interchanged in order to obtain another new parameter vector called the trial vector. To perform the crossover, a new random vector $\left[r_{1}, r_{2}, \cdots, r_{L}\right]$ is generated, where $r_{i}$, for $i=1,2, \cdots, L$, is a uniformly random number chosen from the interval $[0,1]$. In addition, based on this new random vector another binary vector $\left[p_{1}, p_{2}, \cdots, p_{L}\right]$ is derived according to Eq. (9)

$p_{i}=\left\{\begin{array}{ll}1, & \text { if } r_{i}<C R \\ 0, & \text { otherwise }\end{array}\right.$, for $i=1,2, \cdots, L$,

where $C R \in[0,1]$ is the crossover rate and here it is given by 0.5 . Subsequently, the trial vector $W=\left[w_{1}, w_{2} \cdots, w_{L}\right]$ is obtained via the binary vector as Eq. (10)

$w_{i}=\left\{\begin{array}{ll}\theta_{i}, & \text { if } p_{i}=1 \\ v_{i}, & \text { if } p_{i}=0\end{array}\right.$, for $i=1,2, \cdots, L$.

Eq. (10) reveals that the trial vector $W$ is a full crossover result from both $V$ and $\Theta$.

\section{- Selection operation}

The selection operation is a simple mechanism to decide which vector survivals based on their evaluated cost functions. That is, if $C F(W)<C F(\Theta)$, it means that the trial vector $W$ is superior to the target vector $\Theta$, this trial vector remains and replace the target vector. Conversely, the target vector survivals and remove the trial vector in the population. This achieves the selection operation.

It is called a generation or an iteration of the algorithm after executing the above three DE operations once. In this study, if the allowable maximum number of iterations is achieved, the proposed algorithm to solve for parameter estimation problem is terminated. The system block diagram can be schematically depicted in Fig. 2. 




Fig. 2. The proposed system block diagram.

\section{Some simulation results}

This section will show some simulation results for the parameter estimation of Volterra digital system by the developed scheme. The Volterra digital system which is going to be estimated is expressed by Eq. (11)

$$
\begin{aligned}
y[n]=\theta_{1}+\theta_{2} x[n-1] & +\theta_{3} x[n-2]+\theta_{4} x[n-3] \\
& +\theta_{5} x^{2}[n]+\theta_{6} x[n-2] x[n-3],
\end{aligned}
$$

where the unknown system parameters are assumed to be $\theta_{1}=-1.45, \quad \theta_{2}=0.52, \quad \theta_{3}=0.87, \quad \theta_{4}=-1.55$, $\theta_{5}=0.79$, and $\theta_{6}=1.08$, respectively. In the following simulations, the allowable maximum number of iterations and the population size utilized in the algorithm are set to be 200 and 30, and the initial parameter vector is produced from the interval $[-1,1]$ randomly. Besides, the input signal $x[n]$ in Fig. 2 for stimulating both the Volterra digital system and digital model is also randomly generated from $[-1,1]$ for $0 \leq n \leq 100$. After executing the proposed design scheme, some simulation results are obtained. Fig. 3 displays the convergence trajectory of the cost function with respect to the number of iterations during the evolution. The curve almost approximates to zero after performing 50 iterations of the algorithm. In addition, the trajectories of all the estimated system parameters are shown in Fig. 4 with different colors. It is easily seen from Fig. 4 that all the system parameters can be correctly estimated. Corresponding to Fig. 3, these trajectories already approach to correct values after 50 iterations. All simulation results sufficiently prove the effectiveness of the proposed design scheme based on the modified DE algorithm with chaotic random numbers.

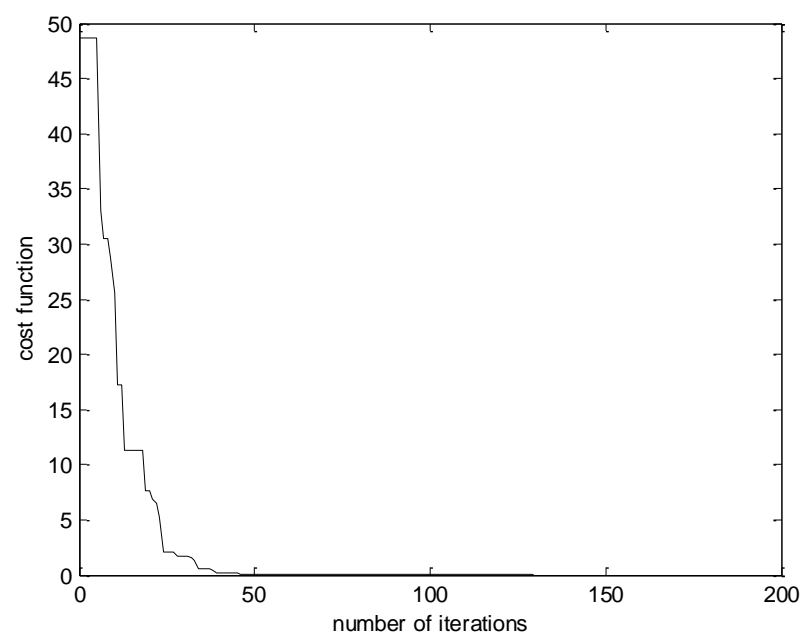

Fig. 3. Convergence trajectory of the cost function.

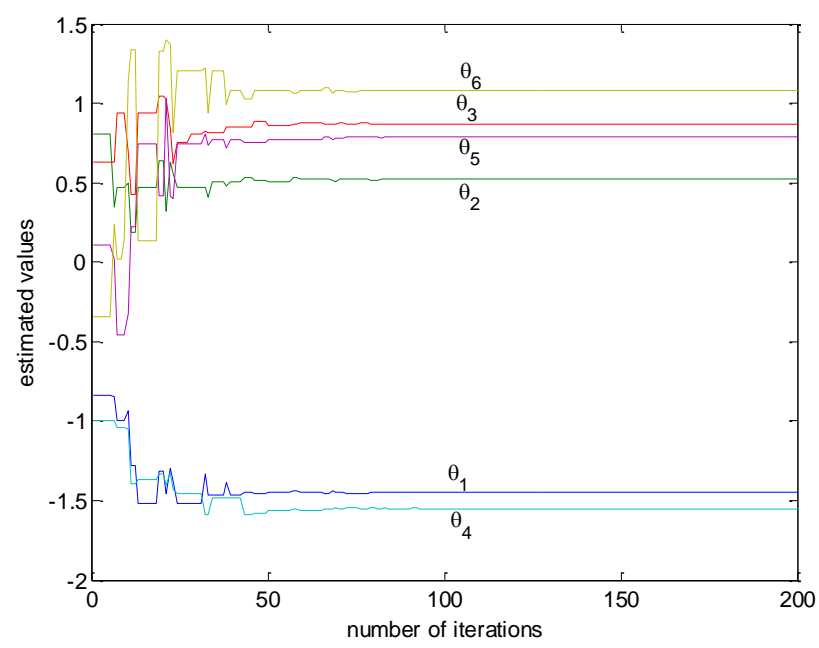

Fig. 4. Convergence trajectories of all the estimated system parameters.

\section{Conclusion and future work}

This paper has successfully presented a new design scheme for the parameter estimation of Volterra digital system. The modified DE algorithm where the chaotic random number is introduced to take place of the fixed mutation constant factor is employed to solve for this estimation problem. A series of chaotic random numbers is produced from the logistic map system. As can be seen from all of simulation results, the proposed method can accurately estimate all the system parameters of Volterra digital filter. In the future work, we will apply the developed method into the control system design. 


\section{Acknowledgment}

This work was supported by the Ministry of Science and Technology of Taiwan under the grant MOST 106-2221-E-366-004.

\section{References}

(1) G. Birpoutsoukis, P.Z. Csurcsia, and J. Schoukens : "Efficient multidimensional regularization for Volterra series estimation", Mechanical Systems and Signal Processing, Vol. 104, pp. 896-914, 2018

(2) O. Karakus, E.E. Kuruoglu, and M.A. Altinkaya : "Bayesian Volterra system identification using reversible jump MCMC algorithm”, Signal Processing, Vol. 141, pp. 125-136, 2017

(3) S. Zhang, J. Zhang, and Y. Pang : "Pipelined set-membership approach to adaptive Volterra filtering", Signal Processing, Vol. 129, pp. 195-203, 2016

(4) J. Prawin, and A. Rama Mohan Rao : "Nonlinear identification of MDOF systems using Volterra series approximation", Mechanical Systems and Signal Processing, Vol. 84, pp. 58-77, 2017

(5) W.D. Chang, C.L. Chi, S.P. Shih, and B.H. Ye : "Parameter estimation algorithms for Volterra digital systems", International Journal of Future Computer and Communication, Vol. 6, pp. 115-118, 2017

(6) Y. Hou, L. Zhao, and H. Lu : "Fuzzy neural network optimization and network traffic forecasting based on improved differential evolution", Future Generation Computer Systems, Vol. 81, pp. 425-432, 2018

(7) S.T. Suganthi, D. Devaraj, K. Ramar, and S. Hosimin Thilagar : "An improved differential evolution algorithm for congestion management in the presence of wind turbin generators", Renewable and Sustainable Energy reviews, Vol. 81, pp. 635-642, 2018

(8) V.C. Mariani, L.d.S. Coelho, and P.K. Sahoo : "Modified differential evolution approaches applied in exergoeconomic analysis and optimization of a cogeneration system", Expert Systems with Applications, Vol. 38, 13886-13893, 2011

(9) J. Kumar, and A.K. Singh : "Workload prediction in cloud using artificial neural network and adaptive differential evolution", Future Generation Computer Systems, Vol. 81, pp. 41-52, 2018

(10) J. Xiao, Y.J. Zhang, and X.K. Xu : "Convergence improvement of differential evolution for community detection in complex networks", Physic A, Vol. 503, pp. 762-779, 2018

(11) W.D. Chang, S.P. Shih, Y.T. Hsiao, Y.T. Xia, T.Y. Liang, and H.T. Huang : "Parameter estimation of bilinear digital systems using differential evolution algorithm", Proceedings of the 3nd International Conference on Intelligent Systems and Image Processing, pp. 54-58, Fukuoka, Japan, 2015

(12)X. Yuan, B. Cao, B. Yang, and Y. Yuan : "Hydrothermal scheduling using chaotic hybrid differential evolution", Energy Conversion and Management, Vol. 49, pp. 3627-3633, 2008 